\title{
Multiple mixing for adele groups and rational points
}

\author{
Alexander Gorodnik ${ }^{1}$ - Ramin Takloo-Bighash ${ }^{2}$. \\ Yuri Tschinkel ${ }^{3,4}$
}

Received: 10 May 2014 / Revised: 20 March 2015 / Accepted: 4 June 2015 /

Published online: 6 August 2015

(C) Springer International Publishing AG 2015

\begin{abstract}
We prove an asymptotic formula for the number of rational points of bounded height on projective equivariant compactifications of $H \backslash G$, where $H$ is a connected simple algebraic group embedded diagonally into $G=H^{n}$.
\end{abstract}

Keywords Rational points $\cdot$ Heights $\cdot$ Mixing $\cdot$ Counting

Mathematics Subject Classification $\quad 14 \mathrm{G} 05 \cdot 11 \mathrm{~F} 70 \cdot 11 \mathrm{G} 50$

Alexander Gorodnik was supported by EPSRC, ERC, and RCUK. Ramin Takloo-Bighash was supported by NSF grant 0701753 . Yuri Tschinkel was partially supported by NSF grants DMS-0739380, 0901777, and 1160859 .

$\bowtie \quad$ Yuri Tschinkel

yuri.tschinkel@googlemail.com; tschinkel@cims.nyu.edu

Alexander Gorodnik

a.gorodnik@bristol.ac.uk

Ramin Takloo-Bighash

rtakloo@math.uic.edu

1 School of Mathematics, University of Bristol, University Walk, Bristol BS8 1TW,

United Kingdom

2 Department of Mathematics, Statistics, and Computer Science, University of Illinois at Chicago, 322 Science and Engineering Offices (M/C 249), 851 S. Morgan Str., Chicago, IL 60607, USA

3 Courant Institute of Mathematical Sciences, New York University, 251 Mercer Str., New York, NY 10012, USA

4 Simons Foundation, 160 Fifth Avenue, New York, NY 10010, USA 


\section{Introduction}

Let $X \subset \mathbf{P}^{n}$ be a smooth projective variety over a number field $F$. Fix a height function

$$
\mathrm{H}: \mathbf{P}^{n}(F) \rightarrow \mathbb{R}_{>0}
$$

and consider the counting function

$$
\mathrm{N}(X, T)=\{x \in X(F): \mathrm{H}(x) \leq T\} .
$$

Manin's conjecture [8] and its refinements by Batyrev-Manin [1], Peyre [17], and Batyrev-Tschinkel [3] predict precise asymptotic formulas for $\mathrm{N}\left(X^{\circ}, T\right)$ as $T \rightarrow$ $\infty$, where $X^{\circ} \subset X$ is an appropriate Zariski open subset of an algebraic variety with sufficiently positive anticanonical class. These formulas involve the following geometric invariants of $X$ :

- the Picard group Pic $(X)$ of $X$;

- the anticanonical class $-K_{X} \in \operatorname{Pic}(X)$;

- the cone of pseudo-effective divisors $\Lambda_{\text {eff }}(X)_{\mathbb{R}} \subset \operatorname{Pic}(X)_{\mathbb{R}}$,

and they depend on an adelic metrization $\mathcal{L}=\left(L,\|\cdot\|_{v}\right)$ of the polarization $L$ giving rise to the embedding $X \subset \mathbf{P}^{n}$, i.e., on a choice of the height function in (1). Given these, one introduces the invariants

$$
a(L), \quad b(L), \quad c(\mathcal{L})
$$

so that the number of $F$-rational points on $X^{\circ}$ of $\mathcal{L}$-height bounded by $T$ is, conjecturally, given by

$$
\mathrm{N}\left(X^{\circ}, \mathcal{L}, T\right)=\frac{c(\mathcal{L})}{a(L)(b(L)-1) !} T^{a(L)} \log (T)^{b(L)-1}(1+\mathrm{o}(1)), \quad T \rightarrow \infty,
$$

see, e.g., [3, Definitions 2.2.4, 2.3.11 and Section 3], for precise definitions of the constants.

These conjectures have stimulated intense research; see $[4,5,16,20]$ for surveys of the current state of this subject. Of particular importance are equivariant compactifications of algebraic groups and their homogeneous spaces. In all equivariant cases considered previously, it was essential that $X$ admits an action, with a dense orbit, of a solvable algebraic group. For example, the paper [19] proves Manin's conjecture for equivariant compactifications of the symmetric space $G \backslash(G \times G)$, a spherical variety. In this paper, we establish these conjectures for a new class of varieties, which includes nonspherical varieties, under a technical assumption on automorphic characters (Assumption 6.1).

Theorem 1.1 Let $H$ be a connected simple algebraic group defined over a number field $F, G=H^{n}$ its $n$-fold product. Let $X$ be a smooth projective $G$-equivariant compactification of $X^{\circ}=H \backslash G$, where $H$ acts on the left diagonally, with boundary $X \backslash X^{\circ}$ a divisor with strict normal crossings. Under Assumption 6.1, X satisfies Manin's conjecture and its refinements, i.e., (2) holds for $L=-K_{X}$. 
This generalizes the case $n=2$ treated in $[10,19]$ to arbitrary $n$. The proof presented here also works, with minor modifications, for semi-simple groups $H$. Compactifications of the homogeneous space $H \backslash H^{n}$ have played an important role in work of L. Lafforgue on the Langlands' conjecture over function fields of curves over finite fields (see, e.g., [13, Chapter 3]). The geometry of these compactifications is surprisingly rich.

Our proof combines ergodic-theoretic methods developed in [11] with geometric integration techniques developed in [6,7]; in particular, it uses neither the theory of height zeta functions nor spectral theory on adelic spaces. On the other hand, it does not allow to establish effective error terms as in the $n=2$ case in [19].

Organization of the paper. In Sects. 2 and 3 we discuss geometric and analytic background and, in particular, establish meromorphic continuation of Igusa-type integrals (Theorem 3.3) that implies an asymptotic formula for volumes of height balls. In Sect. 4, we give a classification of intermediate subgroups $M$ with $H \subset M \subset H^{n}$. This result is used in Sect. 5 where we establish the multiple mixing property for the adelic spaces using measure-rigidity techniques. Finally, our main result is deduced from multiple mixing in Sect. 6.

\section{Geometric background}

Let $F$ be an algebraically closed field of characteristic zero, $G$ a connected semi-simple algebraic group defined over $F$ and $H \subset G$ a connected closed subgroup. Let $X$ be a projective equivariant compactification of $X^{\circ}=H \backslash G$. Throughout, we assume that $X$ is smooth and that the boundary

$$
\bigcup_{\alpha \in \mathcal{A}} D_{\alpha}=X \backslash X^{\circ}
$$

is a divisor with normal crossings, with irreducible components $D_{\alpha}$. We will identify line bundles and divisors with their classes in the Picard group $\operatorname{Pic}(X)$. If $H$ is a parabolic subgroup, then there is no boundary, i.e., $\mathcal{A}$ is empty, and $H \backslash G$ is a generalized flag variety. Distribution of rational points of bounded height on flag varieties was studied in [8]. We will assume that

- $\mathcal{A}$ is not empty,

- $X^{\circ}$ is affine (this holds, e.g., when $H$ is reductive),

- the groups of algebraic characters of $G$ and $H$ are trivial.

Recall that a 1-parameter subgroup of $G$ is a homomorphism $\xi: \mathbb{G}_{m} \rightarrow G$.

Proposition 2.1 Let $G$ be a connected reductive group, $H \subset G$ a closed connected reductive subgroup, and $X$ a smooth projective $G$-equivariant compactification of $X^{\circ}=H \backslash G$. Assume that $G$ and $H$ have no nontrivial algebraic characters. Then

- the classes of irreducible boundary components $D_{\alpha}$ span the Picard group $\operatorname{Pic}(X)_{\mathbb{Q}}$ and the pseudo-effective cone $\Lambda_{\mathrm{eff}}(X) \subset \operatorname{Pic}(X)_{\mathbb{R}}$; 
- the class of the anticanonical line bundle is given by

$$
-K_{X}=\sum_{\alpha \in \mathcal{A}} \kappa_{\alpha} D_{\alpha}
$$

where all $\kappa_{\alpha} \geq 1$.

Proof Fix a polarization $L$ of $X$ and let $X \subset \mathbf{P}^{n}$ be the corresponding projective embedding. After taking a suitable multiple, we may assume that $L$ is $G$-linearized, i.e., the action of $G$ on $X$ extends to an action on the ambient $\mathbf{P}^{n}$ (by [15, Corollary 1.6]). Let $D$ be an effective divisor such that the generic point of $D$ is in $H \backslash G$. There exists a 1-parameter subgroup moving the generic point of $D$. After specializing $D$, at least one of the irreducible components of the limit is supported in the boundary. We can now apply induction on the $L$-degree of the remaining components, if any, to conclude that $D$ is equivalent to an effective divisor with support in the boundary.

On the other hand, the only invertible functions on $H \backslash G$ are constants, by assumption and Rosenlicht's theorem. It follows that there are no relations between classes of the boundary components.

For the second claim, see, e.g., [12, Section 6].

Let $L$ be a big line bundle on $X$. We define

$$
\begin{aligned}
& a(L)=\inf \left\{t \in \mathbb{Q}: t[L]+\left[K_{X}\right] \in \Lambda_{\mathrm{eff}}(X)\right\}, \\
& b(L)=\text { the maximal codimension of the face containing } a(L) L+K_{X} .
\end{aligned}
$$

These invariants depend on the chosen compactification $X$. By Proposition 2.1, we have

$$
L=\sum_{\alpha \in \mathcal{A}} \lambda_{\alpha} D_{\alpha}, \quad \lambda_{\alpha} \in \mathbb{Q}_{>0}
$$

so that the corresponding invariants are given by

$$
a(L)=\max _{\alpha} \frac{\kappa_{\alpha}}{\lambda_{\alpha}}, \quad b(L)=\#\left\{\alpha \in \mathcal{A}: a(L)=\frac{\kappa_{\alpha}}{\lambda_{\alpha}}\right\} .
$$

Remark 2.2 The invariants $a(L)$ and $b(L)$ may be computed even if $X$ is not smooth. Consider an equivariant resolution of singularities $\widetilde{X} \rightarrow X$, and let $\widetilde{L}$ be the pullback of $L$ to $\widetilde{X}$. Put

$$
a(L)=a(\widetilde{L}), \quad b(L)=b(\widetilde{L}) .
$$

A basic result is that this does not depend on the chosen resolution (see, e.g., [12, Section 2]).

The following proposition, used in the proof of Theorem 1.1 in conjunction with results in Sect. 6, has been established in [12]. 
Proposition 2.3 Let $M \subsetneq G$ be a closed connected subgroup containing $H$ and let $Y$ be the closure of $H \backslash M$ in $X$. Then

$$
\left(a\left(-\left.K_{X}\right|_{Y}\right), b\left(-\left.K_{X}\right|_{Y}\right)\right)<\left(a\left(-K_{X}\right), b\left(-K_{X}\right)\right)
$$

in the lexicographic ordering.

Remark 2.4 This fails in the non-equivariant context, see [2] for a counterexample and [3] for a discussion of this "saturation" phenomenon.

\section{Heights and height integrals}

Let $F$ be a number field, $\mathbb{A}$ its ring of adeles, and $\mathbb{A}_{f}$ the subring of finite adeles. Let $v$ be a place of $F$ and $F_{v}$ the corresponding completion; for nonarchimedian $v$ we let $\mathfrak{o}_{v}$ denote the ring of $v$-integers and $\mathfrak{m}_{v}$ its maximal ideal.

Let $X$ be a projective variety over $F, U \subset X$ a Zariski open subset with boundary

$$
\bigcup_{\alpha \in \mathcal{A}} D_{\alpha}=X \backslash U
$$

being a normal crossings divisor. Here $D_{\alpha}$ are $F$-irreducible components, which could be reducible over an algebraic closure $\bar{F}$ of $F$. For each $\alpha$ one can endow the line bundle $\mathcal{O}\left(D_{\alpha}\right)$ with an adelic metric which allows to define local and global heights (see, e.g., [7, Section 2.3]):

$$
\mathrm{H}_{D_{\alpha, v}}: U\left(F_{v}\right) \rightarrow \mathbb{R}_{>0}, \quad \mathrm{H}_{D_{\alpha}}=\prod_{v} \mathrm{H}_{D_{\alpha, v}} .
$$

The heights in (3) give rise to an adelic height system

$$
\begin{aligned}
\bigoplus_{\alpha} \mathbb{C}^{\mathcal{A}} \times U(\mathbb{A}) \stackrel{\mathrm{H}}{\longrightarrow} \mathbb{C} \\
\left(\sum_{\left.s_{\alpha} D_{\alpha},\left(u_{v}\right)\right)} \mapsto \prod_{\alpha} \prod_{v} \mathrm{H}_{D_{\alpha}, v}\left(u_{v}\right)^{s_{\alpha}}\right.
\end{aligned}
$$

which restricts to a Weil height, for $u \in U(F)$ and $\left(s_{\alpha}\right) \in \mathbb{Z}^{\mathcal{A}}$ (see [7, Section 2] for more details).

We will apply this theory in the setup of Sect. 2 . Let $G$ be a connected semisimple algebraic group over $F$ with trivial characters, $H$ a closed connected reductive subgroup, and $X$ a smooth projective $G$-equivariant compactification of the affine variety $X^{\circ}=H \backslash G$ with boundary

$$
\bigcup_{\alpha \in \mathcal{A}} D_{\alpha}=X \backslash G
$$


which we assume to be a divisor with strict normal crossings. The divisors $D_{\alpha}$ can be equipped with an adelic metrization which defines local and global heights on $X^{\circ}(\mathbb{A})$. Furthermore, $G$-equivariance implies that for all but finitely many $v$, the local height functions $\mathrm{H}_{v}$ are right-invariant under $G\left(\mathfrak{o}_{v}\right)$ (see, e.g., [6, Section 3]). The local and global measures $\mathrm{d} \tau_{v}$ and $\mathrm{d} \tau$ coincide with suitably normalized Haar measures $\mathrm{d} x_{v}$ and $\mathrm{d} x$ on $X^{\circ}(\mathbb{A})=(H \backslash G)(\mathbb{A})$.

Lemma 3.1 ([18, Chapter 1, Section 5.4]) Let $G$ be a connected semi-simple algebraic group defined over a field $F$ and $H$ a closed subgroup. Let $X^{\circ}=H \backslash G$ and assume that the map of sets

$$
\mathrm{H}^{1}(F, H) \rightarrow \mathrm{H}^{1}(F, G)
$$

is injective.Then

$$
X^{\circ}(F)=H(F) \backslash G(F) .
$$

Corollary 3.2 Let $H$ be a connected simple algebraic group defined over a field $F$, acting diagonally on $G=H^{n}$. Then

$$
X^{\circ}(F)=H(F) \backslash G(F) .
$$

In particular, if $F$ is a number field, and $F_{v}$ its completion with respect to a place $v$, then

$$
X^{\circ}\left(F_{v}\right)=H\left(F_{v}\right) \backslash G\left(F_{v}\right) \quad \text { and } \quad X^{\circ}(\mathbb{A})=H(\mathbb{A}) \backslash G(\mathbb{A}) .
$$

Proof The map

$$
\mathrm{H}^{1}(F, H) \rightarrow \mathrm{H}^{1}(F, G)
$$

is injective, since $G=H^{n}$ and the map is the diagonal one.

The geometric framework developed in [7, Section 4] allows to establish analytic properties of local and global integrals of the form

$$
\int_{U\left(F_{v}\right)} \mathrm{H}_{v}\left(\mathbf{s}, u_{v}\right)^{-1} \mathrm{~d} \tau_{v}, \quad \int_{U(\mathbb{A})} \mathrm{H}(\mathbf{s}, u)^{-1} \mathrm{~d} \tau,
$$

where $\tau_{v}$ and $\tau$ are certain Tamagawa measures defined in [7, Section 2]. [7, Propositions 4.1.2 and 4.3.5] provide meromorphic continuations for integrals in (5) (see also [19, Theorem 7.1]).

Theorem 3.3 Let $G$ be a connected semi-simple algebraic group and $H \subset G$ a closed subgroup, defined over a number field $F$, satisfying the vanishing condition (4) for $F$ 
and all of its completions. Let $X$ be a smooth projective equivariant compactification of $X^{\circ}=H \backslash G$ with normal crossing boundary $\bigcup_{\alpha \in \mathcal{A}} D_{\alpha}$ and

$$
\mathrm{H}: \mathbb{C}^{\mathcal{A}} \times X^{\circ}(\mathbb{A}) \rightarrow \mathbb{C}
$$

an adelic height system. Then there exists a function $\Phi$, holomorphic and bounded in vertical strips for $\operatorname{Re} s_{\alpha}>\kappa_{\alpha}-\epsilon$, for some $\epsilon>0$, such that for $\mathbf{S}=\left(s_{\alpha}\right)$ in this domain one has

$$
\int_{X^{\circ}(\mathbb{A})} \mathrm{H}(\mathbf{s}, x)^{-1} \mathrm{~d} x=\prod_{\alpha \in \mathcal{A}} \zeta_{F}\left(s_{\alpha}-\kappa_{\alpha}+1\right) \cdot \Phi(\mathbf{s}),
$$

where $\zeta_{F}$ is the Dedekind zeta function.

Applying Tauberian theorems, we obtain the following result.

Lemma 3.4 (well-roundedness of adelic height balls) Let L be a class in the interior of the cone of effective divisors and $\mathrm{H}$ the associated height. Then the corresponding height balls

$$
B_{T}=\left\{x \in X^{\circ}(\mathbb{A}): H(x)<T\right\}
$$

are well-rounded, i.e.,

$$
\lim _{\kappa \rightarrow 1^{+}} \limsup _{T \rightarrow \infty} \frac{\operatorname{vol} B_{\kappa}-\operatorname{vol} B_{\kappa^{-1} T}}{\operatorname{vol} B_{T}}=0 .
$$

Proof This is a corollary of the main theorem of [7, Theorem 1.3], which establishes the claimed asymptotic expansion for $B_{T}$ and well-roundedness in the smooth case. The general case follows from the smooth case: for any constant $c>1$ there exists a smooth metrization such that the corresponding height function $\mathrm{H}^{\prime}$ satisfies

$$
c^{-1} \mathrm{H}^{\prime}<\mathrm{H}<c \mathrm{H}^{\prime}
$$

Thus for any $T>0$, we have

$$
B^{\prime}{ }^{-1} T \subseteq B_{T} \subseteq B^{\prime}{ }_{c T}
$$

so that

$$
\underset{T}{\limsup } \frac{\operatorname{vol} B_{\kappa T}-\operatorname{vol} B_{\kappa^{-1} T}}{\operatorname{vol} B_{T}} \leq \underset{T}{\lim \sup } \frac{\operatorname{vol} B^{\prime}{ }_{c \kappa T}-\operatorname{vol} B^{\prime}{ }_{c^{-1} \kappa^{-1} T}}{\operatorname{vol}{B^{\prime}}_{c^{-1} T}},
$$

which can be made arbitrarily small by taking $c \kappa$ close enough to 1 . This implies that the height balls are well-rounded. 


\section{Intermediate subgroups}

Let $H$ be a connected almost simple algebraic group defined over an algebraically closed field of characteristic zero and $Z(H)$ its center. For $n \in \mathbb{N}$, let $H^{n}$ be the $n$-fold product of $H$ and $\Delta_{n}=H \hookrightarrow H^{n}$ the diagonal. The symmetric group $\mathfrak{S}_{n}$ acts on $H^{n}$ by permution of the coordinates. We call subgroups $M, N$ of $H^{n}$ permutation equal if there is $\sigma \in \mathfrak{S}_{n}$ such that $M=\sigma(N)$. The following proposition is used in the proof of the multiple mixing property in Sect. 5 .

Proposition 4.1 Let $H$ be a connected simple algebraic group and $M$ a connected algebraic group such that

$$
\Delta_{n} \subseteq M \subseteq H^{n}
$$

Then there exist $n_{1}, \ldots, n_{k} \in \mathbb{N}$ such that $\sum_{i=1}^{k} n_{i}=n$ and $M$ is permutation equal to

$$
\Delta_{n_{1}} \times \cdots \times \Delta_{n_{k}}
$$

The remainder of this section is devoted to a proof of Proposition 4.1. The main step is the following version of Goursat's lemma.

Lemma 4.2 Let $\underline{x}_{r}=\left(x_{1}, \ldots, x_{r}\right) \in H^{r}$ be such that for all $i$ and $j \neq i$, we have $x_{i}, x_{i} x_{j}^{-1} \notin Z(H)$. Let $L_{r} \subseteq H^{r}$ be the smallest subgroup containing

$$
\Gamma_{r}=\left\{\left(\delta x_{1} \delta^{-1}, \ldots, \delta x_{r} \delta^{-1}\right): \delta \in H\right\}
$$

Then $L_{r}=H^{r}$.

Proof We assume that $Z(H)=1$ and proceed by induction on $r$. Note that $\Gamma_{1}$ is nontrivial and that it is closed under conjugation so that the closed subgroup of $H$ generated by $\Gamma_{1}$ is normal. Since $H$ is simple, $L_{1}=H$.

For $r>1$. Let $L_{r}$ be the subgroup corresponding to $\underline{x}_{r}=\left(x_{1}, \ldots, x_{r}\right)$, we assume that $L_{r}=H^{r}$. Clearly, $L_{r}$ is the projection of $L_{r+1}$ onto the first $r$ entries. Applying the case $r=1$, we deduce that the projection of $L_{r+1}$ onto the last entry is equal to $H$. Suppose that there is an element $h \in H^{r}$ such that for two distinct elements $u, v \in H$, we have $(h, u) \in L_{r+1}$ and $(h, v) \in L_{r+1}$. Then $\left(e_{r}, u v^{-1}\right) \in L_{r+1}$, where $e_{r}$ denotes the vector in $H^{r}$ consisting of identity elements in every entry. Again by the case when $r=1$, we see that $\left\{e_{r}\right\} \times H \subset L_{r+1}$. Since the projection onto the first $r$ coordinates is surjective, $L_{r+1}=H^{r} \times H$, as required. It remains to rule out the case when for every $h \in H^{r}$ there is a unique $u=u(h)$ such that $(h, u(h)) \in L_{r+1}$. It follows from the uniqueness that the map $\varphi: h \mapsto u(h)$ is a homomorphism $H^{r} \rightarrow H$, and

$$
L_{r+1}=\left\{(h, \varphi(h)): h \in H^{r}\right\} .
$$


Moreover, $\varphi$ is surjective. By construction, if $(h, \varphi(h)) \in L_{r+1}$, then for any $\delta \in H$, we have

$$
\left(\delta_{r} h \delta_{r}^{-1}, \delta \varphi(h) \delta^{-1}\right) \in L_{r+1},
$$

where $\delta_{r}$ denotes the vector in $H^{r}$ with $\delta$ in every entry. It follows from uniqueness that

$$
\varphi\left(\delta_{r} h \delta_{r}^{-1}\right)=\delta \varphi(h) \delta^{-1}
$$

Hence, $\delta^{-1} \varphi\left(\delta_{r}\right)$ commutes with $\varphi(h)$ for every $h \in H^{r}$. Since $\varphi$ is surjective, we see that $\varphi\left(\delta_{r}\right)=\delta$ for every $\delta \in H$.

Set $y=\left(x_{1} x_{r+1}^{-1}, \ldots\right)=\left(y^{\prime}, e\right)$, by definition of $\varphi$, one has $x_{r+1}=\varphi\left(x_{1}, \ldots, x_{r}\right)$, hence $\varphi\left(y^{\prime}\right)=e$. Consequently, $\left(y^{\prime}, e\right) \in L_{r+1}$. Moreover, $y^{\prime} \in H^{r}$ satisfies the condition of the lemma, so that $L_{r+1}$ contains $L_{r} \times\{e\}$. Since the last projection is surjective, this implies $L_{r+1}=H^{r+1}$.

Definition 4.3 Let $r \leq n$ be integers. An admissible embedding of $H^{r}$ in $H^{n}$ is a morphism $\varphi: H^{r} \rightarrow H^{n}$ of the form

$$
\varphi\left(h_{1}, \ldots, h_{r}\right)=\left(h_{i_{1}}, \ldots, h_{i_{n}}\right)
$$

for some integers $i_{1}, \ldots, i_{n} \in\{1, \ldots, r\}$. Up to permutation of coordinates on $H^{n}$, it is of the form

$$
\begin{aligned}
& H^{r} \rightarrow \Delta_{n_{1}} \times \cdots \times \Delta_{n_{r}} \subset H^{n} \\
& \left(h_{1}, \ldots, h_{r}\right) \mapsto(\underbrace{h_{1}, \ldots, h_{1}}_{n_{1}}, \underbrace{h_{2}, \ldots, h_{2}}_{n_{2}}, \ldots, \underbrace{h_{r}, \ldots, h_{r}}_{n_{r}}),
\end{aligned}
$$

with $\sum_{i} n_{i}=n$. An admissible subgroup of $H^{n}$ is the image of an admissible embedding.

Definition 4.4 Given $r \leq n$, we say an element $\underline{x} \in H^{n}$ is of rank $\leq r$, if $\underline{x} \in \iota\left(H^{r}\right)$ for some admissible embedding $\iota$. We say $\underline{x}$ is of rank $r_{0}$, written $r(\underline{x})=r_{0}$, if $r_{0}$ is the smallest number $r$ such that $\underline{x}$ is of rank $\leq r$.

It is clear that for every $\underline{x} \in H^{n}, r(\underline{x}) \leq n$. Note that if $\underline{x} \in H^{n}$ and $\underline{\delta} \in \Delta_{n}$ then

$$
r(\underline{x} \cdot \underline{\delta})=r(\underline{x}), \quad \underline{x} \in H^{n} .
$$

Proof of Proposition 4.1 A reformulation of the statement of the proposition is that if $M$ is a connected subgroup of $H^{n}$ satisfying

$$
\Delta_{n} \subset M \subset H^{n}
$$


then $M$ is admissible. Since the isogeny $\pi: H^{r} \rightarrow \bar{H}^{r}$, where $\bar{H}=H / Z(H)$, defines a bijection between a closed connected subgroup of $H^{r}$ and $\bar{H}^{r}$, it is sufficient to prove the claim assuming that $Z(H)=1$.

Let $r=\max _{\underline{x} \in M} r(\underline{x})$, and let $\underline{x}$ be an element of $M$ which realizes this maximum. As $\Delta_{n} \subset M$, we may assume that no entry of $\underline{x}$ is equal to identity. After rearranging the coordinates, if necessary, we may assume that

$$
\underline{x}=\left(x_{1}, \ldots, x_{1}, x_{2}, \ldots, x_{2}, \ldots, x_{r}, \ldots, x_{r}\right) \in \Delta_{n_{1}} \times \cdots \times \Delta_{n_{r}},
$$

where $x_{i} x_{j}^{-1} \neq e$ for $i \neq j$. Then since $\Delta_{n} \subset M$, it follows from Lemma 4.2 that

$$
N=\Delta_{n_{1}} \times \cdots \times \Delta_{n_{r}} \subseteq M
$$

It suffices to establish that $N=M$. If $M$ were larger than $N$, multiplying a generic element of $N$ by an element of $M \backslash N$ we would get an element $\underline{x}^{\prime}$ with $r\left(\underline{x}^{\prime}\right)>r(\underline{x})$, a contradiction.

\section{Multiple mixing}

Let $H$ be a connected semi-simple algebraic group defined over a number field $F$. The aim of this section is to prove the multiple mixing property for the adelic homogeneous space $Y=H(F) \backslash H(\mathbb{A})$. However, when the group $H$ is not simply connected, $\mathrm{L}^{2}(H(F) \backslash H(\mathbb{A}))$ contains nontrivial one-dimensional representations, and the multiple mixing property holds only on a subset $Y_{W} \subset Y$, which we now introduce. Let $\pi: \widetilde{H} \rightarrow H$ be the universal cover of $H$ and $W$ a compact subgroup of $H(\mathbb{A})$ such that $W \cap H\left(\mathbb{A}_{f}\right)$ is open in $H\left(\mathbb{A}_{f}\right)$. We set

$$
H_{W}=H(F) \pi(\widetilde{H}(\mathbb{A})) W .
$$

By [11, Corollary 4.10], $H_{W}$ is a normal closed co-abelian subgroup of finite index in $H(\mathbb{A})$. We consider the homogeneous space

$$
Y_{W}=H(F) \backslash H_{W},
$$

equipped with the normalized Haar measure dy. Let $\mathrm{C}_{\mathrm{c}}\left(Y_{W}\right)^{W}$ denote the space of continuous compactly supported and $W$-invariant functions on $Y_{W}$.

The following theorem is an adelic version of the multiple mixing of S. Mozes [14].

Theorem 5.1 (multiple mixing) Let $H$ be a connected simple group over $F$ and

$$
\left\{\left(b_{1}^{(n)}, \ldots, b_{r}^{(n)}\right)\right\}_{n \in \mathbb{N}} \subset H_{W}[r]=H_{W} \times \cdots \times H_{W}
$$

a sequence such that for all $i \neq j$,

$$
\lim _{n \rightarrow \infty}\left(b_{i}^{(n)}\right)^{-1} b_{j}^{(n)}=\infty \quad \text { in } H_{W} .
$$


Then for all $f_{1}, \ldots, f_{r} \in \mathrm{C}_{\mathrm{c}}\left(Y_{W}\right)^{W}$, we have

$$
\lim _{n \rightarrow \infty} \int_{Y_{W}} f_{1}\left(y b_{1}^{(n)}\right) \cdots f_{r}\left(y b_{r}^{(n)}\right) \mathrm{d} y=\int_{Y_{W}} f_{1} \mathrm{~d} y \cdots \int_{Y_{W}} f_{r} \mathrm{~d} y .
$$

The proof of Theorem 5.1 is based on an interpretation of integrals in (7) as a sequence of probability measures supported on $Y_{W} \times \cdots \times Y_{W}$ and on an analysis of their limit behaviour using the theory of unipotent flows on adelic spaces developed in [11]. The main technical tools are a partial case of [11, Theorem1.7] combined with the description of intermediate subgroups from Sect. 4.

For $g \in G(\mathbb{A})$ and a measure $v$ on $G(F) \backslash G(\mathbb{A})$, let $g \cdot v$ be the push-forward of $v$ via the right multiplication by $g$.

Theorem 5.2 ([11, Theorem 1.7]) Let G be a connected semi-simple algebraic group defined over a number field $F, H$ a connected semi-simple subgroup defined over $F$, and $V$ a compact subgroup of $G(\mathbb{A})$ such that $V \cap G\left(\mathbb{A}_{f}\right)$ is open in $G\left(\mathbb{A}_{f}\right)$. Let $v_{L}$ be the unique $\widetilde{L}(\mathbb{A})$-invariant probability measure supported on $G(F) \pi(\widetilde{L}(\mathbb{A})) \subset$ $G(F) \backslash G_{V}$, and let $g^{(n)}$ be a sequence in $G(F) \pi(\widetilde{G}(\mathbb{A})) \subset G_{V}$. Then

(a) If the centralizer of $L$ in $G$ is anisotropic over $F$, then the sequence of measures $\left\{g^{(n)} \cdot v_{L}\right\}$ is precompact in the weak* topology.

(b) Suppose that a probability measure $\mu$ on $G(F) \backslash G_{V}$ is a limit of the sequence $\left\{g^{(n)} \cdot v_{L}\right\}$ in the weak $k^{*}$ topology. Then there exist a connected algebraic subgroup $M$ of $G$ over $F$ and sequences $\left\{\delta^{(n)}\right\} \subset G(F),\left\{l^{(n)}\right\} \subset \pi(\widetilde{L}(\mathbb{A}))$ such that

- $\delta^{(n)} L\left(\delta^{(n)}\right)^{-1} \subset M$,

- $\delta^{(n)} l^{(n)} g^{(n)} \rightarrow g \in \pi(\widetilde{G}(\mathbb{A}))$,

and the limit measure $\mu$ can be described as follows: there is a normal subgroup $M_{0} \subset M(\mathbb{A})$ of finite index, containing $M(F) \pi(\widetilde{M}(\mathbb{A}))$, such that for all $f \in$ $\mathrm{C}_{\mathrm{c}}\left(G(F) \backslash G_{V}\right)^{V}$,

$$
\int_{G(F) \backslash G_{V}} f \mathrm{~d} \mu=\int_{G(F) \backslash G_{V}} f \mathrm{~d}\left(g \cdot v_{M_{0}}\right),
$$

where $v_{M_{0}}$ denotes the unique invariant probability measure supported on $G(F) M_{0} \subset G(F) \backslash G_{V}$.

Proof of Theorem 5.1 We apply Theorem 5.2 to the groups

$$
G=H^{r}=H \times \cdots \times H, \quad L=\Delta_{r}=\{(h, \ldots, h): h \in H\}, \quad V=W \times \cdots \times W .
$$

Since $H(F) \pi(\widetilde{H}(\mathbb{A}))$ is a normal subgroup of $H_{W}$ (see [11, Section 4]) and $W$ is compact, the normalized Haar measure on $Y_{W}$ can be written as

$$
\int_{Y_{W}} f \mathrm{~d} y=\int_{Y_{W} \times W} f(u w) \mathrm{d} v_{H}(u) \mathrm{d} w, \quad f \in \mathrm{C}_{\mathrm{c}}\left(Y_{W}\right),
$$


where $v_{H}$ is the unique $\widetilde{H}(\mathbb{A})$-invariant probability measure on $H(F) \pi(\widetilde{H}(\mathbb{A})) \subset Y_{W}$, and $\mathrm{d} w$ is the probability invariant measure on $W$. Therefore,

$$
\int_{Y_{W}} f_{1}\left(x b_{1}^{(n)}\right) \cdots f_{r}\left(x b_{r}^{(n)}\right) \mathrm{d} x=\int_{Y_{W} \times W} f_{1}\left(u w b_{1}^{(n)}\right) \cdots f_{r}\left(u w b_{r}^{(n)}\right) \mathrm{d} v_{H}(u) \mathrm{d} w .
$$

If we show that for every fixed $w \in W$, we have

$$
\lim _{n \rightarrow \infty} \int_{Y_{W}} f_{1}\left(u w b_{1}^{(n)}\right) \cdots f_{r}\left(u w b_{r}^{(n)}\right) \mathrm{d} v_{H}(u)=\int_{Y_{W}} f_{1} \mathrm{~d} y \cdots \int_{Y_{W}} f_{r} \mathrm{~d} y,
$$

then the theorem would follow from the Lebesgue dominated convergence theorem.

We write $w b_{i}^{(n)}=s_{i}^{(n)} w_{i}^{(n)}$ for $s_{i}^{(n)} \in H(F) \pi(\widetilde{H}(\mathbb{A}))$ and $w_{i}^{(n)} \in W$. Since the functions $f_{i}$ are assumed to be $W$-invariant,

$$
\int_{Y_{W}} f_{1}\left(u w b_{1}^{(n)}\right) \cdots f_{r}\left(u w b_{r}^{(n)}\right) \mathrm{d} v_{H}(u)=\int_{Y_{W}} f_{1}\left(u s_{1}^{(n)}\right) \cdots f_{r}\left(u s_{r}^{(n)}\right) \mathrm{d} v_{H}(u) .
$$

Since $W$ is compact, we have

$$
\left(s_{i}^{(n)}\right)^{-1} s_{j}^{(n)}=w_{j}^{(n)} \cdot\left(b_{i}^{(n)}\right)^{-1} b_{j}^{(n)} \cdot\left(w_{j}^{(n)}\right)^{-1} \rightarrow \infty
$$

for all $i \neq j$. We set

$$
s^{(n)}=\left(s_{1}^{(n)}, \ldots, s_{r}^{(n)}\right) \in G(F) \pi(\widetilde{G}(\mathbb{A})) .
$$

Then

$$
\int_{Y_{W}} f_{1}\left(u s_{1}^{(n)}\right) \cdots f_{r}\left(u s_{r}^{(n)}\right) \mathrm{d} v_{H}(u)=\int_{G(F) \backslash G_{V}}\left(f_{1} \otimes \cdots \otimes f_{l}\right) \mathrm{d}\left(s^{(n)} \cdot v_{L}\right) .
$$

Now it remains to determine the limit points of the sequence of measures $s^{(n)} \cdot v_{L}$ in the weak* topology. We first note that the centraliser of $L$ in $G$ is equal to $Z(H) \times \cdots$ $\times Z(H)$. Hence, by Theorem $5.2(\mathrm{a})$, the sequence of measures $s^{(n)} \cdot v_{L}$ is precompact. Let $\mu$ be a probability measure on $G(F) \backslash G_{V}$ which is a limit point of this sequence. The measure $\mu$ is described by in Theorem 5.2(b). In particular, we obtain that there exist a connected algebraic subgroup $M$ of $G$ and a sequence $\delta^{(n)} \in G(F)$ such that

$$
L \subseteq\left(\delta^{(n)}\right)^{-1} M \delta^{(n)} \subseteq G
$$

From the classification of intermediate subgroups in Proposition 4.1, we deduce that

$$
M=\delta^{(n)} N_{n}\left(\delta^{(n)}\right)^{-1},
$$

where $N_{n}$ is an admissible subgroup (in the sense of Definition 4.3). 
We claim that $M=G$. Indeed, suppose that $M \subsetneq G$. Since the number of admissible subgroups is finite, we may assume, after passing to a subsequence, that $N_{n}=N \subsetneq G$ is independent of $n$. Then there exist indices $i \neq j$ such that for the corresponding projection map $\pi_{i j}: G \rightarrow H \times H$, we have $\pi_{i j}(N)=\Delta$, where $\Delta$ denotes the diagonal subgroup in $H \times H$. Let $\delta=\delta^{(1)}$ and $\sigma^{(n)}=\delta^{-1} \delta^{(n)}$. Since

$$
\delta^{(1)} N\left(\delta^{(1)}\right)^{-1}=\delta^{(n)} N\left(\delta^{(n)}\right)^{-1},
$$

we obtain

$$
\pi_{i j}\left(\sigma^{(n)}\right) \Delta \pi_{i j}\left(\sigma^{(n)}\right)^{-1}=\Delta
$$

and

$$
\left(1,\left(\sigma_{i}^{(n)}\right)^{-1} \sigma_{j}^{(n)}\right) \Delta\left(1,\left(\sigma_{i}^{(n)}\right)^{-1} \sigma_{j}^{(n)}\right)=\Delta
$$

This implies that

$$
z_{n}=\left(\sigma_{i}^{(n)}\right)^{-1} \sigma_{j}^{(n)} \in Z(H)
$$

By Theorem 5.2(b), we also know that there exist $l^{(n)} \in \pi(\widetilde{L}(\mathbb{A}))$ such that the sequence $\delta^{(n)} l^{(n)} S^{(n)}$ converges. Then the sequence $\sigma^{(n)} l^{(n)} S^{(n)}$ converges too, and in particular,

$$
\left(\sigma_{i}^{(n)} l_{i}^{(n)} s_{i}^{(n)}\right)^{-1}\left(\sigma_{j}^{(n)} l_{j}^{(n)} s_{j}^{(n)}\right)
$$

converges. Since $l_{i}^{(n)}=l_{j}^{(n)}$ and $z_{n} \in Z(H)$, we obtain

$$
\begin{aligned}
\left(\sigma_{i}^{(n)} l_{i}^{(n)} s_{i}^{(n)}\right)^{-1}\left(\sigma_{j}^{(n)} l_{j}^{(n)} s_{j}^{(n)}\right) & =\left(s_{i}^{(n)}\right)^{-1}\left(l_{i}^{(n)}\right)^{-1}\left(\sigma_{i}^{(n)}\right)^{-1} \sigma_{j}^{(n)} l_{j}^{(n)} s_{j}^{(n)} \\
& =\left(s_{i}^{(n)}\right)^{-1}\left(l_{i}^{(n)}\right)^{-1} z_{n} l_{j}^{(n)} s_{j}^{(n)} \\
& =z_{n}^{-1}\left(s_{i}^{(n)}\right)^{-1} s_{j}^{(n)}
\end{aligned}
$$

Since $z_{n}$ runs over the finite set $Z(H)$, it follows that $\left(s_{i}^{(n)}\right)^{-1} s_{j}^{(n)}$ converges, which is a contradiction. This proves that $M=G$.

By the last statement of Theorem 5.2, there is a finite index subgroup $M_{0} \subseteq$ $M(\mathbb{A})=G(\mathbb{A})$, containing $G(F) \pi(\widetilde{G}(\mathbb{A}))$, and $g \in \pi(\widetilde{G}(\mathbb{A}))$ such that for all $f \in \mathrm{C}_{\mathrm{c}}\left(G(F) \backslash G_{V}\right)^{V}$,

$$
\int_{G(F) \backslash G_{V}} f \mathrm{~d} \mu=\int_{G(F) \backslash G_{V}} f \mathrm{~d}\left(g \cdot v_{M_{0}}\right) .
$$

Since $G(F) \pi(\widetilde{G}(\mathbb{A}))$ is a normal coabelian subgroup of $G_{V}$ (see [11, Section 4]), $M_{0}$ is also normal coabelian. As in (8), the normalized Haar measure $\mathrm{d} z$ on $G(F) \backslash G_{V}$ is given by 


$$
\int_{G(F) \backslash G_{V}} f \mathrm{~d} z=\int_{G(F) \backslash G_{V} \times V} f(u v) \mathrm{d} v_{M_{0}}(u) \mathrm{d} v, \quad f \in \mathrm{C}_{\mathrm{c}}\left(G(F) \backslash G_{V}\right),
$$

where $\mathrm{d} v$ is the normalized Haar measure on $V$. For $f \in \mathrm{C}_{\mathrm{c}}\left(G(F) \backslash G_{V}\right)^{V}$, using that $M_{0}$ is coabelian, we obtain

$$
\begin{aligned}
\int_{G(F) \backslash G_{V}} f \mathrm{~d} z & =\int_{G(F) \backslash M_{0} \times V} f(u v g) \mathrm{d} v_{M_{0}}(u) \mathrm{d} v \\
& =\int_{G(F) \backslash M_{0} \times V} f(u g v) \mathrm{d} v_{M_{0}}(u) \mathrm{d} v=\int_{G(F) \backslash G_{V}} f \mathrm{~d}\left(g \cdot v_{M_{0}}\right) .
\end{aligned}
$$

This proves that every limit point of the sequence $g^{(n)} \cdot v_{L}$ is a probability measure which is equal to $\mathrm{d} z$ on $\mathrm{C}_{\mathrm{c}}\left(G(F) \backslash G_{V}\right)^{V}$. The proof of Theorem 5.1 is complete.

\section{Counting rational points}

Let $H$ be a connected simple algebraic group defined over a number field $F, G=H^{r}$, and $X$ be a smooth projective equivariant compactification of $X^{\circ}=H \backslash G$, where $H$ is embedded diagonally. Let $L$ be a line bundle on $X$ such that its class is in the interior of the cone of effective divisors $\Lambda_{\text {eff }}(X)$. By Proposition 2.1, we can write

$$
L=\sum_{\alpha \in \mathcal{A}} \lambda_{\alpha} D_{\alpha}, \quad \lambda_{\alpha} \in \mathbb{Q}_{>0}
$$

Let

$$
\mathrm{H}=\mathrm{H}_{\mathcal{L}}: X^{\circ}(F) \rightarrow \mathbb{R}_{>0}
$$

be a height corresponding to a smooth metrization of $L$ as in Sect. 3 (or in [7, Section 2.1]).

The adelic height function $\mathrm{H}$ is invariant under a compact open subgroup $V$ of $G\left(\mathbb{A}_{f}\right)$, and we may assume that $V=W \times \cdots \times W$ for a compact open subgroup $W$ of $H\left(\mathbb{A}_{f}\right)$. From now on, we make the following assumption.

Assumption 6.1 There are no nontrivial automorphic characters of $G(\mathbb{A})$ invariant under $V$.

This assumption simplifies the analysis of volume asymptotics of adelic height balls, via Tauberian theorems applied to Igusa integrals. It holds, e.g., in the following cases (see [19, Proposition 2.1 and Remark 2.2]):

- if $G$ is a connected semi-simple simply connected group,

- if $G$ is split and of adjoint type over a number field of class number 1 , and $\mathrm{H}$ is invariant under $\prod_{v \nmid \infty} G\left(\mathfrak{o}_{v}\right)$. 
Example 6.2 Consider $G=\mathrm{PGL}_{2}$ over a field $F$ of class number 2. Let $E / F$ be the Hilbert class field and $\omega_{E / F}$ the corresponding quadratic character. Then

$$
\omega_{E / F} \circ \text { det }: \operatorname{PGL}_{2}\left(\mathbb{A}_{F}\right) \rightarrow \pm 1
$$

is an unramified nontrivial automorphic character.

We define the subgroups $G_{V} \subset G(\mathbb{A})$ and $H_{W} \subset H(\mathbb{A})$ as in (6). The homogeneous space

$$
X_{V}=H_{W} \backslash G_{V}
$$

naturally embeds into $X^{\circ}(\mathbb{A})$ as an open subset, and by Corollary 3.2,

$$
X^{\circ}(\mathbb{A}) \simeq H(\mathbb{A}) \backslash G(\mathbb{A})
$$

We equip $X^{\circ}(\mathbb{A})$ with the Tamagawa measures $\mathrm{d} x$, defined as in [7, Section 2]. The regularization of the measure as in [7] requires vanishing of cohomology $\mathrm{H}^{i}\left(X, \mathcal{O}_{X}\right)$, for $i=1,2$, which follows in our case by general vanishing arguments, as $-K_{X}$ is big (by [9, Theorem 1.2]); it is also evident from the explicit volume computation in Lemma 3.4.

Define the height balls in $X_{V}$ by

$$
B_{T}=B_{T, \mathcal{L}}=\left\{x \in X_{V}: \mathrm{H}_{\mathcal{L}}(x)<T\right\} .
$$

Lemma 6.3 Assume that the line bundle $L$ is in the interior of the effective cone. Then

$$
\operatorname{vol} B_{T}=c(\mathcal{L}) \cdot T^{a(L)} \log (T)^{b(L)-1}(1+\mathrm{o}(1)) \quad \text { as } \quad T \rightarrow \infty
$$

with $c(\mathcal{L})>0$ and $a(L), b(L)$ as in (2).

Proof Using a standard Tauberian argument (see, for instance, [7]), it suffices to show that

$$
\mathbf{Z}(s)=\int_{X_{V}} \mathrm{H}(x)^{-s} \mathrm{~d} x
$$

has an isolated pole at $a(L)$ of order $b(L)$ and that it admits a meromorphic continuation to $\operatorname{Re} s>a(L)-\epsilon$, for some $\epsilon>0$. We recall (see [11, Section 4]) that, in general, $G_{V}$ is a normal closed coabelian subgroup of $G(\mathbb{A})$. Under Assumption 6.1, it coincides with $G(\mathbb{A})$. It remains to invoke Theorem 3.3.

Definition 6.4 Let $X$ be an equivariant compactification of $X^{\circ}=H \backslash G$ and $H^{\prime} \subset G$ any closed proper subgroup containing the diagonal, i.e., $H \subset H^{\prime}$. Let $X^{\prime} \subsetneq X$ be the induced equivariant compactification of $H^{\prime}$. A line bundle $L$ on $X$ is called balanced with respect to $H^{\prime}$ if 


$$
\left(a\left(\left.L\right|_{X^{\prime}}\right), b\left(\left.L\right|_{X^{\prime}}\right)\right)<(a(L), b(L)),
$$

in the lexicographic ordering. It is called balanced if this property holds for every such $H^{\prime} \subsetneq G$.

If $X^{\prime}$ is not smooth, the invariants are computed on an equivariant resolution of singularities (see Remark 2.2).

Remark 6.5 This property fails in simple examples: $X=\mathbf{P}^{3} \times \mathbf{P}^{3}$ considered as an equivariant compactification of $\mathbb{G}_{m}^{6}$ or $\mathbb{G}_{a}^{6}$, or $\mathrm{PGL}_{2} \times \mathrm{PGL}_{2}$, with $L=\left(\lambda_{1}, \lambda_{2}\right)$ and $\lambda_{1} \neq \lambda_{2}$.

Lemma 6.6 Assume that the line bundle $L$ is balanced. Then, for every smooth adelic metrization of $L$, every compact subset $K$ of $H_{W}$ and $i \neq j$, one has

$$
\frac{\operatorname{vol}\left(B_{T} \cap\left\{\left(x_{1}, \ldots, x_{n}\right) \in H \backslash G: x_{i}^{-1} x_{j} \in K\right\}\right)}{\operatorname{vol} B_{T}} \rightarrow 0 \quad \text { as } \quad T \rightarrow \infty .
$$

Proof Let $M \subset G=H^{n}$ be the subgroup defined by $x_{i}=x_{j}$. Lemma 3.4 implies that, for $T \rightarrow \infty$, one has

$$
\begin{aligned}
\operatorname{vol} B_{T} & =c T^{a(X, L)} \log (T)^{b(X, L)-1}(1+\mathrm{o}(1)), \\
\operatorname{vol}\left(B_{T} \cap\left\{x_{i}^{-1} x_{j}=1\right\}\right) & =c^{\prime} T^{a\left(Y,\left.L\right|_{Y}\right)} \log (T)^{b\left(Y,\left.L\right|_{Y}\right)-1}(1+\mathrm{o}(1)),
\end{aligned}
$$

where $Y$ is the induced equivariant compactification of

$$
Y^{\circ}=(H \backslash M) \subset(H \backslash G)=X^{\circ} \subset X
$$

and

$$
a(L), \quad b(L), \quad \text { resp. } \quad a\left(\left.L\right|_{Y}\right), \quad b\left(\left.L\right|_{Y}\right)
$$

are the geometric invariants defined in Sect. 2. When $L$ is balanced, (9) follows, by definition.

Let $K \subset G(\mathbb{A})$ be a compact subset. Consider translates $M_{k}$ of $M$ by $k \in K$. The asymptotic of

$$
\operatorname{vol}\left(B_{T} \cap\left\{\left(x_{1}, \ldots, x_{n}\right) \in H \backslash G: x_{i}^{-1} x_{j}=k\right\}\right)
$$

is determined by analytic properties of the height integral

$$
I(\mathbf{s}, k)=\int_{Y^{\circ}(\mathbb{A})} \mathrm{H}(\mathbf{s}, y k)^{-1} \mathrm{~d} y=\prod_{v} \int_{Y^{\circ}\left(F_{v}\right)} \mathrm{H}_{v}\left(\mathbf{s}, y_{v} k_{v}\right)^{-1} \mathrm{~d} y_{v},
$$

where $Y^{\circ}=H \backslash M$ and $\mathrm{d} y, \mathrm{~d} y_{v}$ are suitably normalized Haar measures. Note that the adelic function

$$
k \mapsto \mathrm{H}(\mathbf{s}, y k),
$$


is continuous, with $\mathrm{H}_{v}\left(\mathbf{s}, y_{v} k_{v}\right)=\mathrm{H}_{v}\left(\mathbf{s}, y_{v}\right)$ for all but finitely many $v$. Specialize the integral $I(\mathbf{s}, k)$ to $\mathbf{s}=s L$. We know that each local integral

$$
\int_{Y^{\circ}\left(F_{v}\right)} \mathrm{H}_{v}\left(s L, y_{v} k_{v}\right)^{-1} \mathrm{~d} y_{v}
$$

is holomorphic for $\operatorname{Re} s>a\left(\left.L\right|_{Y}\right)-\epsilon$, for some $\epsilon>0$, and that the Euler product $I(s L, k)$ has an isolated pole at $s=a=a\left(\left.L\right|_{Y}\right)$ of order $b=b\left(\left.L\right|_{Y}\right)$. When $L$ is balanced, (9) holds for translates $M_{k}$.

Moreover, the function

$$
k \mapsto(s-a)^{b} \cdot I(s L, k)
$$

is uniformly continuous and nonvanishing, for $\operatorname{Re} s>a-\epsilon$, since only finitely many $v$ are affected and the local integrals vary uniformly continuously with $k$. We conclude that

$$
s \mapsto \int_{K} I(s L, k) \mathrm{d} k
$$

has an isolated pole at $s=a$ of order $b$. It follows that, for $T \rightarrow \infty$,

$$
\begin{aligned}
\operatorname{vol}\left(B_{T} \cap\left\{x_{i}^{-1} x_{j} \in K\right\}\right) & =\int_{K} \operatorname{vol}\left(B_{T} \cap\left\{x_{i}^{-1} x_{j}=k\right\}\right) \mathrm{d} k \\
& =c T^{a} \log (T)^{b-1}(1+\mathrm{o}(1)),
\end{aligned}
$$

with some constant $c=c(\mathcal{L})>0$.

Remark 6.7 If the height function is not balanced, the proper subvariety defined by

$$
\left\{x_{i}^{-1} x_{j}=\text { constant }\right\}
$$

contributes a positive proportion of rational points to the asymptotic. This is an example of the saturation phenomenon observed in [3], cf. Remark 2.4.

As a corollary of Theorem 5.1 we obtain an equidistribution on the space $Z_{V}=$ $G(F) \backslash G_{V}$. We denote by $\mathrm{d} y$ and $\mathrm{d} z$ the normalized Haar measures supported on $Y_{W}=H(F) \backslash H_{W}$ and $Z_{V}=G(F) \backslash G_{V}$ respectively. Let $\mathrm{d} x$ denote the restriction of the Tamagawa measure on $X_{V}$. We consider $Y_{W}$ as a subspace of $Z_{V}$ embedded in $Z_{V}$ diagonally.

Corollary 6.8 If the line bundle $L$ is balanced, then for every $f \in \mathrm{C}_{\mathrm{c}}\left(Z_{V}\right)$,

$$
\lim _{T \rightarrow \infty} \frac{1}{\operatorname{vol} B_{T}} \int_{B_{T}} \mathrm{~d} y \int_{Y_{W}} f(y x) \mathrm{d} x=\int_{Z_{V}} f \mathrm{~d} z .
$$


Proof By the Stone-Weierstrass theorem, it suffices to consider functions of the form $f=f_{1} \otimes \cdots \otimes f_{n}$ with $f_{i} \in \mathrm{C}_{\mathrm{c}}\left(Y_{W}\right)$. In this case,

$$
I(x)=\int_{Y_{W}} f(y x) \mathrm{d} y=\int_{Y_{W}} f_{1}\left(y x_{1}\right) \cdots f_{r}\left(y x_{r}\right) \mathrm{d} y .
$$

Since $B_{T}$ is invariant under $V=W \times \cdots \times W$,

$$
\int_{B_{T}} I(x) \mathrm{d} x=\int_{B_{T}} \int_{Y_{W}} \bar{f}_{1}\left(y x_{1}\right) \cdots \bar{f}_{r}\left(y x_{r}\right) \mathrm{d} y \mathrm{~d} x
$$

where $\bar{f}_{i}(y)=\int_{W} f_{i}(y w) \mathrm{d} w$, where $\mathrm{d} w$ denotes the normalized Haar measure on $W$. Hence, we may assume that functions $f_{i}$ are $W$-invariant.

Given a compact subset $K$ of $H_{W}$, we set

$$
B_{T, K}=\left\{x \in B_{T}: x_{i}^{-1} x_{j} \notin K, i \neq j\right\} .
$$

By Theorem 5.1, for every $\epsilon>0$, there exists a compact subset $K$ of $H_{W}$ such that for all $x=\left(x_{1}, \ldots, x_{r}\right) \in B_{T, K}$, we have

$$
\left|I(x)-\int_{Y_{W}} f_{1} \mathrm{~d} y \cdots \int_{Y_{W}} f_{r} \mathrm{~d} y\right|<\epsilon
$$

and

$$
\int_{B_{T, K}} I(x) \mathrm{d} x=\operatorname{vol} B_{T, K} \int_{Y_{W}} f_{1} \mathrm{~d} y \cdots \int_{Y_{W}} f_{r} \mathrm{~d} y+\mathrm{O}\left(\epsilon \operatorname{vol} B_{T, K}\right) .
$$

Also,

$$
\int_{B_{T} \backslash B_{T, K}} I(x) \mathrm{d} x=\mathrm{O}\left(\operatorname{vol}\left(B_{T} \backslash B_{T, K}\right)\right) .
$$

Since the line bundle is balanced, it follows from Lemma 6.6 that

$$
\frac{\operatorname{vol}\left(B_{T} \backslash B_{T, K}\right)}{\operatorname{vol} B_{T}} \rightarrow 0 \quad \text { as } \quad T \rightarrow \infty
$$

Hence, combining (10) and (11), we deduce that

$$
\limsup _{T \rightarrow \infty}\left|\frac{1}{\operatorname{vol} B_{T}} \int_{B_{T}} I(x) \mathrm{d} x-\int_{Y_{W}} f_{1} \mathrm{~d} y \cdots \int_{Y_{W}} f_{r} \mathrm{~d} y\right|=\mathrm{O}(\epsilon)
$$

for every $\epsilon>0$, which proves the corollary. 
From Corollary 6.8, we deduce

Theorem 6.9 Let $H$ be a connected simple algebraic group over $F, G=H^{n}$, and $X$ a G-equivariant compactification of $X^{\circ}=H \backslash G$. Let $H_{W} \subseteq H(\mathbb{A})$ be the normal subgroup of finite index defined in (6). Let $L$ be a balanced line bundle on $X$. Then

$$
\begin{aligned}
\left|X^{\circ}(F) \cap B_{T}\right| & =\operatorname{vol}\left(H(F) \backslash H_{W}\right)^{1-r} \cdot \operatorname{vol} B_{T} \cdot(1+\mathrm{o}(1)), \\
& =c \operatorname{vol}\left(H(F) \backslash H_{W}\right)^{1-r} \cdot T^{a(L)} \log (T)^{b(L)-1}(1+\mathrm{o}(1)),
\end{aligned}
$$

as $T \rightarrow \infty$, where $c=c(\mathcal{L})$ is as in Lemma 6.3.

Proof Let $\mathrm{d} h$ be the Tamagawa measure on $H(\mathbb{A})$ restricted to $H_{W}$. Then the Haar measure $\mathrm{d} g$ on $G_{V}$ can be written as

$$
\int_{G_{V}} \tilde{f} \mathrm{~d} g=\int_{X_{V}} \mathrm{~d} x \int_{H_{W}} \widetilde{f}(h x) \mathrm{d} h, \quad \widetilde{f} \in \mathrm{C}_{\mathrm{c}}\left(G_{V}\right) .
$$

Take $\kappa>1$, and let $U$ be a symmetric neighborhood of identity in $G_{V}$ such that

$$
B_{T} \cdot U \subset B_{\kappa} T \quad \text { for all } T
$$

Let $\tilde{f} \in \mathrm{C}_{\mathrm{c}}\left(G_{V}\right)$ be a nonnegative function with supp $\tilde{f} \subset U$ and $\int_{G_{V}} \tilde{f} \mathrm{~d} g=1$. Put

$$
f(g)=\sum_{\gamma \in G(F)} \widetilde{f}\left(\gamma^{-1} g\right)
$$

Then, for every $x \in X_{V}$,

$$
\int_{Y_{W}} f(y x) \mathrm{d} y=\frac{1}{\operatorname{vol}\left(H(F) \backslash H_{W}\right)} \sum_{\gamma \in H(F) \backslash G(F)} \int_{H_{W}} \tilde{f}\left(\gamma^{-1} h x\right) \mathrm{d} h .
$$

If $x \in B_{\kappa^{-1} T}$ and $\gamma^{-1} h x \in U$, then $\gamma \in h x U$; using (12) we have $\gamma \in B_{T}$, since $B_{T}$ consists of cosets of $H_{W}$ and $h \in H_{W}$. Hence, (13) implies that

$$
\begin{aligned}
\operatorname{vol}\left(H(F) \backslash H_{W}\right) \int_{B_{\kappa^{-1} T}} \mathrm{~d} x & \int_{Y_{W}} f(y x) \mathrm{d} y \\
& =\sum_{\gamma \in H(F) \backslash G(F) \cap B_{T}} \int_{H_{W} \times B_{\kappa^{-1} T}} \tilde{f}\left(\gamma^{-1} h x\right) \mathrm{d} h \mathrm{~d} x \\
& \leq \sum_{\gamma \in H(F) \backslash G(F) \cap B_{T}} \int_{G_{V}} \tilde{f}\left(\gamma^{-1} g\right) \mathrm{d} g \\
& =\left|H(F) \backslash G(F) \cap B_{T}\right| .
\end{aligned}
$$


If $\gamma \in B_{T}$ and $\gamma^{-1} h x \in U$, then $x \in h^{-1} \gamma U$; using (12) we have $x \in B_{\kappa T}$. Now (13) implies that

$$
\begin{aligned}
\left|H(F) \backslash G(F) \cap B_{T}\right| & =\sum_{\gamma \in H(F) \backslash G(F) \cap B_{T}} \int_{G_{V}} \tilde{f}\left(\gamma^{-1} g\right) \mathrm{d} g \\
& =\sum_{\gamma \in H(F) \backslash G(F) \cap B_{T}} \int_{H_{W} \times X_{V}} \tilde{f}\left(\gamma^{-1} h x\right) \mathrm{d} h \mathrm{~d} x \\
& \leq \sum_{\gamma \in H(F) \backslash G(F)} \int_{H_{W} \times B_{\kappa} T} \tilde{f}\left(\gamma^{-1} h x\right) \mathrm{d} h \mathrm{~d} x \\
& =\operatorname{vol}\left(H(F) \backslash H_{W}\right) \int_{B_{\kappa} T} \mathrm{~d} x \int_{Y_{W}} f(y x) \mathrm{d} y .
\end{aligned}
$$

By Lemma 6.3,

$$
\lim _{T \rightarrow \infty} \frac{\operatorname{vol} B_{\kappa T}}{\operatorname{vol} B_{T}}=\kappa^{a(L)}
$$

Combining (14) with Corollary 6.8, we obtain

$$
\begin{aligned}
\liminf _{T \rightarrow \infty} & \frac{\left|H(F) \backslash G(F) \cap B_{T}\right|}{\operatorname{vol} B_{T}} \\
& \geq \lim _{T \rightarrow \infty} \frac{\operatorname{vol} B_{\kappa^{-1} T}}{\operatorname{vol} B_{T}} \cdot \lim _{T \rightarrow \infty} \frac{\operatorname{vol}\left(H(F) \backslash H_{W}\right)}{\operatorname{vol} B_{\kappa^{-1} T}} \int_{B_{\kappa^{-1} T}} \mathrm{~d} x \int_{Y_{W}} f(y x) \mathrm{d} y \\
& =\kappa^{-a(L)} \operatorname{vol}\left(H(F) \backslash H_{W}\right) \int_{Z_{V}} f \mathrm{~d} z \\
& =\kappa^{-a(L)} \frac{\operatorname{vol}\left(H(F) \backslash H_{W}\right)}{\operatorname{vol}\left(G(F) \backslash G_{V}\right)}=\kappa^{-a(L)} \operatorname{vol}\left(H(F) \backslash H_{W}\right)^{1-r} .
\end{aligned}
$$

Similarly, it follows from (15) that

$$
\limsup _{T \rightarrow \infty} \frac{\left|H(F) \backslash G(F) \cap B_{T}\right|}{\operatorname{vol} B_{T}} \leq \kappa^{a(L)} \operatorname{vol}\left(H(F) \backslash H_{W}\right)^{1-r} .
$$

Since these estimates hold for all $\kappa>1$, we conclude that

$$
\left|H(F) \backslash G(F) \cap B_{T}\right|=\operatorname{vol}\left(H(F) \backslash H_{W}\right)^{1-r} \operatorname{vol} B_{T} \cdot(1+\mathrm{o}(1))
$$

as $T \rightarrow \infty$. Since $X^{\circ}(F)=H(F) \backslash G(F)$ by Corollary 3.2, this proves the first part of the theorem. The second part follows from Lemma 6.3.

Theorem 1.1 follows by applying Proposition 2.3, which insures that the anticanonical line bundle $-K_{X}$ is balanced. 
Acknowledgments We are grateful to Antoine Chambert-Loir, Amos Nevo, and Brendan Hassett for useful comments and suggestions. We are especially grateful to the referee who carefully read the paper and made a number of valuable suggestions.

\section{References}

1. Batyrev, V.V., Manin, Yu.I.: Sur le nombre des points rationnels de hauteur borné des variétés algébriques. Math. Ann. 286(1-3), 27-43 (1990)

2. Batyrev, V.V., Tschinkel, Yu.: Rational points on some Fano cubic bundles. C. R. Acad Sci. Paris Sér. I Math. 323(1), 41-46 (1996)

3. Batyrev, V.V., Tschinkel, Yu.: Tamagawa numbers of polarized algebraic varieties. In: Peyre, E. (ed.) Nombre et Répartition de Points de Hauteur Bornée (Paris, 1996), Astérisque, vol. 251, pp. 299-340. Société Mathématique de France, Paris (1998)

4. Browning, T.D.: Quantitative Arithmetic of Projective Varieties. Progress in Mathematics, vol. 277. Birkhäuser, Basel (2009)

5. Chambert-Loir, A.: Lectures on height zeta functions: at the confluence of algebraic geometry, algebraic number theory, and analysis. In: Bhowmik, G., Matsumoto, K., Tsumura, H. (eds.) Algebraic and Analytic Aspects of Zeta Functions and $L$-functions. Mathematical Society of Japan Memoirs, vol. 21, pp. 17-49. Mathematical Society of Japan, Tokyo (2010)

6. Chambert-Loir, A., Tschinkel, Yu.: On the distribution of points of bounded height on equivariant compactifications of vector groups. Invent. Math. 148(2), 421-452 (2002)

7. Chambert-Loir, A., Tschinkel, Yu.: Igusa integrals and volume asymptotics in analytic and adelic geometry. Conflu. Math. 2(3), 351-429 (2010)

8. Franke, J., Manin, Yu.I., Tschinkel, Yu.: Rational points of bounded height on Fano varieties. Invent. Math. 95(2), 421-435 (1989)

9. Fu, B., Zhang, D.-Q.: A characterization of compact complex tori via automorphism groups. Math. Ann. 357(3), 961-968 (2013)

10. Gorodnik, A., Maucourant, F., Oh, H.: Manin's and Peyre's conjectures on rational points and adelic mixing. Ann. Sci. Éc. Norm. Supér. 41(3), 383-435 (2008)

11. Gorodnik, A., Oh, H.: Rational points on homogeneous varieties and equidistribution of adelic periods. Geom. Funct. Anal. 21(2), 319-392 (2011)

12. Hassett, B., Tanimoto, S., Tschinkel, Yu.: Balanced line bundles and equivariant compactifications of homogeneous spaces (2013). arXiv:1307.5467 (to appear in Int. Math. Res. Not. IMRN)

13. Lafforgue, L.: Chirurgie des Grassmanniennes. CRM Monograph Series, vol. 19. American Mathematical Society, Providence (2003)

14. Mozes, S.: Mixing of all orders of Lie groups actions. Invent. Math. 107(2), 235-241 (1992)

15. Mumford, D., Fogarty, J., Kirwan, F.: Geometric Invariant Theory. Ergebnisse der Mathematik und ihrer Grenzgebiete, vol. 34, 3rd edn. Springer, Berlin (1994)

16. Oh, H.: Orbital counting via mixing and unipotent flows. In: Einsiedler, M.L., et al. (eds.) Homogeneous Flows, Moduli Spaces and Arithmetic. Clay Mathematics Proceedings, vol. 10, pp. 339-375. American Mathematical Society, Providence (2010)

17. Peyre, E.: Hauteurs et mesures de Tamagawa sur les variétés de Fano. Duke Math. J. 79(1), 101-218 (1995)

18. Serre, J.-P.: Cohomologie Galoisienne. Lecture Notes in Mathematics, vol. 5, 5th edn. Springer, Berlin (1994)

19. Shalika, J., Takloo-Bighash, R., Tschinkel, Yu.: Rational points on compactifications of semi-simple groups. J. Amer. Math. Soc. 20(4), 1135-1186 (2007)

20. Tschinkel, Yu.: Algebraic varieties with many rational points. In: Darmon, H., Ellwood, D.A., Hassett, B., Tschinkel, Yu. (eds.) Arithmetic Geometry. Clay Mathematics Proceedings, vol. 8, pp. 243-334. American Mathematical Society, Providence (2009) 